\title{
Value of radiotherapy for tumour on the bronchial stump after resection for bronchial carcinoma
}

\author{
MR LAW, JM HENK, SC LENNOX, ME HODSON \\ From the Brompton Hospital and Cardiothoracic Institute and the Royal Marsden Hospital, London
}

\begin{abstract}
Two groups have been selected from 1000 patients who had lobectomy or pneumonectomy for bronchial carcinoma. The first group was of 26 patients with tumour affecting the mucosa of the resected bronchial margin reported histologically at the time of surgery. Twelve of these were given postoperative radiotherapy but with no apparent benefit, either in the incidence of recurrence of tumour at the bronchial stump or in five-year survival. The second group was of 17 patients who developed recurrence of tumour at the bronchial stump, bronchoscopically confirmed, some time after surgery. In six of these cases the recurrence was detected while it was confined to the bronchial stump region, and these patients were then given radiotherapy. Five of the six survived five years after radiotherapy, with complete eradication of the tumour recurrence confirmed by repeat bronchoscopy. In 11 cases the tumour recurrence was not detected until it had become more extensive. Radiotherapy may also have prolonged survival in some of these patients, although none survived five years.
\end{abstract}

The value of radiotherapy in prolonging survival of patients with tumour on the bronchial stump after surgical resection for bronchial carcinoma has never been shown convincingly. A review is reported of the effect of radiotherapy in two clinically different types of case: (a) postoperative patients with reported microscopic tumour of the mucosa of the resected bronchial margin, and $(b)$ patients with macroscopic recurrence of tumour in the bronchial stump, detected bronchoscopically some time after surgery.

\section{Patients and methods}

The records of 1000 patients who had undergone lobectomy or pneumonectomy for bronchial carcinoma during 1966-75 were examined. Two series of cases (not mutually exclusive) were selected.

The first series consisted of 26 cases with tumour of the mucosa of the resected bronchial margin reported histologically at the time of surgery (excluding carcinoma in situ). Some clinicians routinely gave postoperative radiotherapy to such patients; as a consequence of this policy 12 of the 26 patients were treated by external megavoltage

Address for reprint requests: Dr MR Law, Brompton Hospital, Fulham Road, London SW3. radiotherapy (4200-5000 rads). The interval between surgery and the commencement of radiotherapy was from one to three months; the duration of radiotherapy was from four to six weeks.

The policy of other clinicians was not to irradiate such patients, so that the remaining 14 patients did not receive radiotherapy. These non-irradiated patients have been used as a control group. No patient in either the irradiated or the non-irradiated group had evidence of distant metastases at presentation or evidence of residual tumour on the postoperative chest radiograph. Patients with evidence of extrapulmonary spread or lymph-node metastasis at surgery, or with histological evidence of tumour invasion of the outer wall of the resected bronchial margin, were not included. All tumours were squamous-cell carcinomas.

The second series consisted of 17 patients who had presented several months or years after surgery with recurrence of tumour in the bronchial stump (table). These cases underwent bronchoscopy; the recurrence was in all cases evident macroscopically and confirmed histologically by biopsy. The tumour recurrence was seen to be confined to the bronchial stump region at bronchoscopy in six of the 17 cases, but in 11 there was extension from the bronchial stump along the mucosa, affecting the main bronchus (in patients who had lobectomy), trachea, or contralateral main bronchus. 
Survival of patients according to the extent of recurrence of tumour at bronchial stump

\begin{tabular}{|c|c|c|c|c|}
\hline \multirow{2}{*}{$\begin{array}{l}\text { Extent of recurrence } \\
\text { at bronchoscopy }\end{array}$} & \multirow{2}{*}{$\begin{array}{l}\text { No } \\
\text { of } \\
\text { patients }\end{array}$} & \multirow{2}{*}{$\begin{array}{l}\text { Interval between surgery } \\
\text { and confirmation of } \\
\text { recurrence (months) }\end{array}$} & \multicolumn{2}{|c|}{ Survival after bronchoscopy (months before death) } \\
\hline & & & With irradiation & Without irradiation \\
\hline Confined to stump region & 6 & $\begin{array}{l}23 \\
13,26,29,32,40\end{array}$ & $\begin{array}{l}4 \\
\text { All five patients* } \\
\text { apparently } \\
\text { cured (see text) }\end{array}$ & \\
\hline $\begin{array}{l}\text { Extending into main bronchus } \\
\text { (lobectomy cases) }\end{array}$ & 4 & $58,35,27,18$ & $15,16,22,32$ & \\
\hline Extending to lateral tracheal wall & 5 & $\begin{array}{l}13,52,41 \\
24,35\end{array}$ & $12, \dagger 33,46$ & 4,8 \\
\hline $\begin{array}{l}\text { Extending into contralateral } \\
\text { main bronchus }\end{array}$ & 2 & 81 & 11 & $\begin{array}{l}4,0 \\
10\end{array}$ \\
\hline
\end{tabular}

*Two of whom had radioactive gold grains implanted.

$\dagger$ This patient had radioactive gold grains implanted.

In seven of these 17 patients tumour on the resected bronchial margin had been detected histologically at the time of surgery; these seven cases were therefore included in the first series also. Three of the seven patients had received postoperative radiotherapy. Tumour had not been detected on the resected bronchial margin in the remaining 10 patients. No case had evidence of lymph-node metastasis or invasion of mediastinal structures by tumour at surgery. These 17 patients had all presented with recent haemoptysis but no other symptoms, and had no radiographic evidence of tumour recurrence. They had all been cigarette smokers, but stopped smoking at the time of their original presentation. All tumours were squamous-cell carcinomas in this series also. Pneumonectomy had been performed in four cases, lobectomy in nine, and lobectomy with sleeve resection of the main bronchus in four.

Fourteen patients were irradiated after confirmation of the recurrence-three by bronchoscopic implantation of radioactive gold grains and 11 by external megavoltage radiotherapy (5000-6100 rads (50-61 Gy) over five to seven weeks). Three, in whom the recurrent tumour was more extensive, were not irradiated.

\section{Results}

\section{POSTOPERATIVE RADIOTHERAPY}

In the series of 26 cases with tumour of the resected bronchial margin reported histologically at the time of surgery, five of the 12 irradiated patients and four of the 14 non-irradiated patients survived five years. The difference was not significant. Three of the 12 irradiated and four of the 14 non-irradiated cases developed subsequent macroscopic stump recurrence of tumour. No patient in either group developed a bronchopleural fistula.
RADIOTHERAPY FOR SUBSEQUENT BRONCHIAL STUMP RECURRENCE OF TUMOUR

The survival of the 17 patients with bronchoscopically proved recurrence of tumour at the bronchial stump is shown in the table.

The six patients in whom tumour was detected when confined to the bronchial stump region were all given radiotherapy. One died of distant metastases after only four months. Of the remaining five, three are still alive, having survived five, eight, and 10 years from bronchoscopic confirmation of tumour recurrence. Repeat bronchoscopy after radiotherapy showed no macroscopic evidence of tumour in the bronchial stump, and biopsies gave negative results. The other two died of heart disease after five years, with no evidence of tumour recurrence at necropsy.

Of the 11 patients whose recurrent tumour was more extensive at the time of bronchoscopy, the three non-irradiated ones all survived less than a year, while survival in the eight irradiated patients was from 11 to 46 months (table). Repeat bronchoscopy in four of these patients showed partial regression of the recurrent tumour after irradiation. Complete regression occurred in one patient with the trachea affected, who died of distant metastases after 46 months with no bronchoscopic evidence of tumour recurrence around the stump shortly before death.

Three patients developed a recurrent tumour at the bronchial stump despite postoperative radiotherapy. One, with a recurrent tumour of limited extent, was treated by implantation of radioactive gold grains and is alive after five years. One patient received further external radiotherapy but survived only 16 months, and the third had extensive recurrence, was not further irradiated, and survived 10 months.

Three of the 14 irradiated patients were treated 
by implantation of radioactive gold grains into the tumour. Two of the patients who survived five years were treated in this way. In the third case the tumour had extended to affect the trachea. It was found to have become considerably smaller on repeat bronchoscopy after implantation of gold grains; the patient, however, died with distant metastases shortly afterwards.

The interval between surgery and confirmation of tumour recurrence at the bronchial stump bore no apparent relation to survival after confirmation of recurrence (table).

\section{Discussion}

Radiotherapy has not previously been shown to be effective in the treatment of macroscopic recurrence of carcinoma in the bronchial stump. It has been claimed, however, that some cases of bronchial carcinoma are potentially curable by radiotherapy, and the published reports of five-year survival after radical radiotherapy for limited disease have recently been summarised. ${ }^{1}$ In the present study five patients survived for at least five years after detection of recurrence of carcinoma at the bronchial stump. In these five the tumour recurrence had been confined to the stump region, and was radically irradiated. Subsequent bronchoscopy indicated total eradication of the recurrent tumour.

The value of postoperative radiotherapy for residual tumour has not been clearly determined. ${ }^{23}$ It did not appear to have been of benefit in the patients tabulated by Jeffery. ${ }^{4}$ Guttmann irradiated 20 patients after incomplete resection of bronchial carcinoma, including four with the bronchial margin affected, without apparent benefit. ${ }^{5}$ In the present study the five-year survival rate and the rate of subsequent recurrence of tumour at the bronchial stump in cases of histologically reported tumour of the mucosa of the resected bronchial margin were similar in patients given postoperative radiotherapy and in non-irradiated patients. Although the study was retrospective, a degree of randomisation was present in that the selection of patients to be irradiated was determined by the policies of different clinicians; no differences in prognostic features were apparent between the two groups. But despite the fact that all cases from a 10-year period were included, there were too few to demonstrate or refute a small benefit from postoperative radiotherapy. Possibly the irradiated patients who later presented with recurrence at the bronchial stump were those with biologically unfavourable tumours. A disadvantage of routine postoperative radiotherapy is that histologically reported tumour on the resected bronchial margin does not necessar- ily herald subsequent recurrence of tumour; prolonged survival of such patients has been reported, ${ }^{24}$ and in the present study only four of the 14 nonirradiated patients later developed recurrence at the bronchial stump. Moreover, of those who do develop subsequent recurrence of tumour at the bronchial stump, some may be salvaged by radiotherapy after diagnosis of the recurrence.

Irradiation of macroscopic recurrent tumour when confined to the bronchial stump region was clearly of value. It is uncertain whether radiotherapy prolonged survival in the eight cases where the recurrent tumour had become more extensive. The longer-surviving patients (who lived 32, 33, and 46 months) may have benefited from radiotherapy; complete eradication of a recurrent tumour at the stump that extended to the trachea was seen in the patient who survived 46 months before dying of distant metastases.

Treatment of tumour recurrence by implantation of gold grains appeared at least as effective as external radiotherapy, and did not produce the side effects of radiotherapy.

Detection of tumour recurrence at the bronchial stump at an early stage improved the results of radiotherapy. But only six of the 17 patients with recurrence at the bronchial stump presented with haemoptysis while the tumour recurrence remained confined to the stump region; in the remaining 11 it had become more extensive before haemoptysis occurred. Of the 17 patients, all had proximal tumours; four had lobectomy with sleeve resection of the main bronchus, and in seven there was a histology report of tumour at the bronchial margin at the time of surgery. Routine bronchoscopy of selected patients at regular intervals might lead to detection of tumour recurrence at the bronchial stump at an earlier stage, when radiotherapy might be curative. Haemoptysis is a clear indication for bronchoscopy in a patient in whom recurrence of tumour at the bronchial stump is a possibility.

The radiotherapy was carried out under the supervision of the late Dr RL Morgan. We would like to express our thanks to the physicians and surgeons of the Brompton Hospital, whose patients we studied, and to Miss S Hockley for secretarial assistance.

\section{References}

'Phillips TL, Miller RJ. Should asymptomatic patients with
inoperable bronchogenic carcinoma receive immediate
radiotherapy? Yes. Am Rev Respir Dis 1978;117:405-10.
2 Shields TW. The fate of patients after incomplete resection of
bronchial carcinoma. Surg Gynecol Obstet 1974;139:569-72., 
${ }^{3}$ Deeley TJ. The treatment of carcinoma of the bronchus. $\mathrm{Br} J$ Radiol 1967;40:801-22.

4 Jeffery RM. Tumour remaining in the bronchial stump following resection. Ann R Coll Surg Engl 1972;51:55-9.
${ }^{3}$ Guttmann RJ. Results of radiation therapy in patients with inoperable carcinoma of the lung whose status was established at exploratory thoracotomy. Am J Roentgenol Radium Ther Nucl Med 1965;93:99-103. 